\title{
IMAGE ET MÉMOIRE
}

\author{
Adriano Duarte Rodrigues ${ }^{1}$
}

Prenons une photo de mes vacances de l'année dernière. Il est évident que ce ne sont pas mes vacances ni un de leurs moments que je retrouve sur la photo que je contemple maintenant, malgré ce que suggère le langage courant. Ce que je vois sur cette photo n'est que l'effet ou le reflet d'un moment de mes vacances, fixé sur une pellicule exposée pendant un certain laps de temps à la lumière. Cet effet ou ce reflet, je le reconnais comme la représentation de certains traits d'un instant qui a été prélevé du flux des journées qui se sont écoulées l'année dernière, lors de mes vacances. Comment se produit cette reconnaissance de l'effet produit, dans le cas de l'image de mes vacances, prise par un dispositif technique comme celui de l'appareil photographique ou d'une caméra ? Est-ce le même processus qui est à l'œuvre dans les cas des images mentales et des images du discours?

Dès 1889, dans l'Essai sur les Données Immédiates de la Conscience, Bergson faisait la distinction entre deux sens de l'expérience : d'un côté, l'expérience de la durée, de ce flux continu de la conscience, et de l'autre l'expérience monothétique, constituée d'unités discrètes que l'on découpe de ce flux et que l'on représente après coup, grâce à l'activité réfléchissante ${ }^{2}$. Il attirait ainsi l'attention sur la nature ouverte des relations que la conscience entretient avec le flux continu

1 Professeur au Département de Linguistique de la Faculté des Sciences Sociales et Humaines de l'Université Nouvelle de Lisbonne

2 H. BeRgSON, Essai sur les Données Immédiates de la Conscience, Paris, PUF, 2007, pp. 56-104.

Recherches en communication, $\mathrm{n}^{\circ} 29$ (2008). 
de l'expérience au sein duquel nous sommes immergés ou pris par la poursuite de nos activités journalières. Lors de l'action en cours nous pouvons imaginer le but à atteindre ou des motivations qui nous amènent à l'entreprendre ${ }^{1}$, mais ce n'est que après l'avoir finie que nous sommes en mesure d'en former des images, à partir des matériaux prélevés par l'imagination du flux que la mémoire fait revenir au présent.

C'est donc bien un processus anamnésique qui est à l'œuvre dans la formation des images. La mémoire fait revenir au présent des moments notables découpés du flux de l'expérience passée, moments dont l'imagination fixe des instantanés. L'enregistrement de ces moments à l'aide d'un dispositif technique, qu'il s'agisse d'un appareil photographique, d'une caméra ou d'un dessin, exige que l'on sorte du flux continu de la conscience et des activités poursuivies et que l'on découpe des instants ou des unités discrètes de l'ensemble continu de la durée.

Je vais essayer de déceler la manière dont la mémoire joue par rapport à la représentation et par rapport au sens de chacune des modalités de l'image, des images mentales, des images fixées par un dispositif technique et des images du discours.

\section{Mémoire et représentation}

Etant donné sa nature analogique, la reconnaissance ou l'identification du référent d'une image dépend d'un processus cognitif fort complexe. Il a la particularité de faire jouer la mémoire ou de faire intervenir le souvenir d'expériences avec lesquels l'image établit des rapports. De ce point de vue général, l'image, quelle qu'en soit d'ailleurs la nature ou le support, réveille des résonances cachées, enfouies dans des strates de l'expérience, faisant ainsi émerger à la conscience et rendre notables des moments qu'elle sélectionne et fixe. Nous pouvons dire que la conscience les fait donc retourner, dans le double sens de ce terme, en tant qu'elle les fait revenir au présent et en tant qu'elle les montre à l'envers. Elle les fait revenir, dans la mesure où elle nous dévoile ou nous fait apercevoir ce qui nous avait échappé lors d'une perception originaire, étant donné la manière fluide et polythétique comme elle nous était alors apparue. Elle les montre à l'envers,

1 Je fais ici allusion à la distinction que Alfred Schütz faisait entre "motifs en vue de" (Um-zu-Motiv) et "motifs parce que" (Weil-Motiv). A. SCHÜTZ, The Phenomenology of the Social World, Northwestern University Press 1967, pp. 8696). 
une fois qu'elle les retire du cadre originaire de la subjectivité pour les mettre à distance, pour les objectiver, à partir d'un point de vue adopté par l'imagination. Il faut encore une fois rappeler la distinction que Bergson faisait entre le continuum de la conscience des expériences présentes et la nature fragmentaire et discrète de l'expérience réflexive que je peux obtenir, au présent, en image de mes expériences passées ${ }^{1}$. L'image n'est, de ce point de vue, que le reflet produit par la capacité de réflexion et par l'imagination, la cristallisation d'unités discrètes qu'elle découpe du continuum de mes expériences passées. Mais, ceci dit, il faut insister sur ce retournement comme effet de la conversion de l'expérience subjective, vécue comme immersion du sujet dans le fleuve de la durée, dans une expérience objectivée par le travail que l'imagination, produit après coup, grâce aux matériaux que la mémoire met à sa disposition.

Certes, l'image entretient avec le réel un rapport analogique, mais elle ne dévoile son référent que dans la mesure où je peux distinguer ou discriminer le segment de réalité représentée parmi d'autres segments avec lesquels elle le met en rapport. De son côté, le verbal n'est pas entièrement de l'ordre du digital ; il relève également de l'analogique, sa référence ne pouvant être saisie que si je peux appréhender l'image du monde auquel il se réfère ou qu'il désigne. La composante digitale d'un discours seule ne saurait être suffisante pour constituer son référent ni déployer son sens, n'étant qu'un agencement de matériaux sonores qui n'accèderaient même pas au statut de signifiants. Il ne suffit donc pas de dire que la représentation des images est analogique et que la représentation verbale est digitale. Il faut aussi rendre compte de ce qui demeure de digital dans l'analogique et de ce qui demeure d'analogique dans le digital. La représentation des images est analogique, mais elle dépend d'un travail de discrimination de l'expérience. La représentation verbale est digitale, mais je ne peux la percevoir que si elle s'ouvre à une réalisation imaginaire de son référent.

C'est bien donc le produit de l'imagination que le dispositif technique fixe et objective une fois pour toutes ${ }^{2}$. Ce qui distingue l'image que je contemple, et qui est le produit d'un dispositif technique de médiatisation, des images produites, soit par la contemplation du

1 H. BERGSON, op. cit., pp. 37, 67-68, 95-98,142,158 et 176.

2 C'est donc de manière appropriée et tout à fait suggestive que nous qualifions d'objectif le système optique d'un appareil de prise de vue ou de projection qui assure la formation d'une image. 
monde réel, soit par l'écoute ou la lecture d'un discours, n'est donc pas la distinction entre la représentation analogique et la représentation digitale, mais l'effet produit par le dispositif qui assure la réalisation de son référent. Ainsi, le dispositif technique fait que l'image que je contemple offre à ma contemplation un référent immanent objectivé, tandis que ma perception sensorielle du monde réel dévoile une réalité transcendante, puisqu'elle laisse indéfiniment différée sa perspective ou son cadre, sa saisie étant donc inévitablement limitée par la face que les choses offrent à ou tournent vers mon regard, cadre qui se déplace à chaque fois que je la vise.

Le référent du discours, de son côté, est le produit du dispositif de l'énonciation, faisant intervenir l'interaction des énoncés avec l'ensemble des instances de l'énonciation ou l'expérience intersubjective, actuelle ou virtuelle, du cadre énonciatif. Les images du discours sont ainsi inséparables de l'expérience du langage qui éveille, dans la conscience, un monde intersubjectif qui fait revenir des images d'expériences passées qui se situent au sein de ce monde. Il faut sans doute souligner à ce propos la consistance du monde intersubjectif, fait de rapports matérialisés sous le mode de normes et d'expectatives ${ }^{1}$.

Nous voyons ainsi que l'image fixée par un dispositif technique procède d'un travail de fixation de la perspective ou du cadre du référent, travail qui, dans le cas des images du discours, est le produit du travail du dispositif énonciatif, tandis que, dans le cas de l'image mentale, est entièrement réalisé par l'imagination.

\section{La mémoire et le sens de l'image}

Si tous les animaux sont doués de la capacité d'identification ou de reconnaissance d'images, puisqu'ils sont doués de conscience, seul l'être humain est en mesure de leur assigner du sens.

On aurait tendance à parler du sens de l'image comme s'il s'agissait d'une idée ou d'un concept à laquelle elle renverrait. C'est une manière impropre de parler, puisqu'elle confond la signification avec le sens. Une image n'a pas, à proprement parler, de signification, puisqu'elle ne renvoie pas à un concept, n'étant pas le produit d'un travail de conceptualisation, mais de l'imagination. Il faut insister sur ce point pour reconnaître que l'image procède de la volonté ou du désir de rendre

1 A. SCHÜTZ, op. cit., p. 115 et pp. 139-207. 
présente une expérience absente, qui m'échappe dans sa durée indéfiniment différée, et non pas d'un travail de l'intelligence, qui cherche à rendre compte de ce qui est, à embrasser ou à immobiliser, à fixer le réel par une saisie schématique.

Mais l'image n'a pas non plus de sens en elle-même. C'est moi qui lui assigne un sens chaque fois que je la contemple. Elle prend donc autant de sens que de fois qu'elle s'offre à ma visée contemplative, parce que c'est de ces visées, toujours particulières et éphémères, que des sens lui adviennent, les sens concrets et particuliers auxquels je la destine lors de ma saisie contemplative de ses formes.

Cette visée contemplative particulière est constitutive du sens des images, parce qu'elle dirige leur perception dans une certaine orientation, leur faisant jouer un certain rôle dans un monde délimité par les interactions que je leur fais établir avec d'autres images lors de leur contemplation. Ainsi la photo que je tiens devant moi peut prendre le sens d'une photo de mes vacances, parce que précisément j'établis, ici et maintenant, un rapport de ce qu'elle représente avec d'autres images qu'elle fait retourner au présent, peu importe d'ailleurs que ces autres images soient mentales et me reviennent lors de sa remémoration ou qu'elles soient fixées sur un support matériel quelconque, comme dans le cas d'autres images d'un film ou que je peux retrouver sur un album de photos.

Ce n'est que de cette interaction concrète à laquelle je la destine qu'une image prend un sens pour moi. En dehors de ce rapport elle reste énigmatique ou, si l'on préfère, elle a un sens potentiel, en attente de pouvoir retrouver d'autres images avec lesquelles je puisse la faire interagir. Certes, il se peut que je trouve une photo par hasard sur mon bureau qui ne me dit rien, que je ne peux pas mettre en rapport avec d'autres images. Je ne dis évidemment pas qu'elle est insensée ou dépourvue de sens, mais énigmatique, menant éventuellement à une sorte de travail de détective, travail de réactivation de la mémoire en vue de retrouver dans le flux de l'expérience passée d'autres fragments avec lesquels est en rapport le fragment représenté et dont il enregistre et fixe la trace.

C'est pourquoi le sens de l'image est toujours inséparable d'un travail de narrativisation, effective ou potentielle. C'est ce travail d'enchaînement du fragment représenté aux autres fragments, découpés par la mémoire, du flux de l'expérience passée et dont je peux rendre compte par un récit, qui lui attribue une destinée, l'oriente dans une certaine direction, lui donne un sens. 


\section{La nature interactive des différentes modalités d'images}

La distinction entre les différentes modalités d'images, entre les images mentales, que j'obtiens à partir de la remémoration du monde réel, les images fixées par un dispositif technique sur support matériel et les images du discours, va maintenant nous aider à comprendre les différentes modalités d'interaction qui me conduisent à leur attribuer du sens lors de leur contemplation.

Les modalités d'interaction des images du discours ainsi que celles qui sont fixées sur support matériel ont en commun de dépendre du fonctionnement d'un dispositif. C'est donc de la nature et du mode de fonctionnement de ces dispositifs que nous devons rendre compte si nous voulons comprendre le sens que je fais jouer aux images qu'ils constituent et fixent. Je ne suis en mesure d'assigner un sens à une image fixée sur un support matériel ou à une image du discours que si je peux reconnaître les frontières qui délimitent leur cadre ou leur monde imagétique.

Appelons hors-champ l'ensemble des images avec lesquelles une image fixée sur support matériel interagit et qui se situent à l'intérieur de ces frontières. Dans le cas des images du discours, c'est l'ensemble des présuppositions de son dispositif énonciatif qui joue un rôle identique à celui que joue le hors-champ dans le cas des images produites par un dispositif technique.

C'est faute de pouvoir reconnaître ce monde présupposé qui se trouve au sein du cadre ou à l'intérieur des frontières du monde dans lequel je situe une image que je n'arrive pas à lui assigner un sens précis. Ainsi, par exemple, comprendre le regard de la jeune fille que j'identifie sur une photo ou sur l'écran revient à mettre en rapport ce regard, soit avec un objet quelconque dont je me souviens, mais qui n'est pourtant pas représenté sur l'image, soit avec mon propre regard.

Dans le cas où ce regard se dirige vers un objet hors-champ dont je me souviens, j'occupe ma place transcendante de spectateur responsable pour l'assignation du sens de l'image que je contemple. Mais dans le cas où c'est mon regard que le regard de la jeune fille fixe, ma place se dédouble. J'occupe alors, à la fois, la place transcendante responsable de l'assignation du sens et une place immanente au sein du monde imagétique pour y jouer le rôle de hors-champ. En un certain sens, je ne vois pas seulement l'image; je suis aussi constitué comme hors-champ, comme objet d'un regard avec lequel l'image que je contemple inte- 
ragit, se formant une sorte de boucle entre le regard qui regarde et le regard regardé.

Mais que veut dire être regardé par le regard représenté sur une image? Ça ne veut certainement pas dire que je suis regardé comme objet d'une interaction intentionnelle unique, qui me choisit parmi d'autres objets d'interaction possibles. Cela veut dire que, en contemplant l'image, je me place à l'endroit que le regard représenté sur l'image a constitué comme endroit virtuel d'interaction.

C'est donc en situant comme hors-champ un fragment de l'expérience que l'imagination découpe et met à ma disposition que le dispositif technique constitue les conditions d'assignation d'un sens. Etre en mesure d'assigner du sens à une image relève d'une compétence proprement imagétique de mise en rapport des formes représentées avec des fragments de monde auquel elles appartiennent.

C'est ce rapport ambivalent entre la scène qui se déroule au sein de la représentation fixée par le dispositif et ce qui, bien que non représenté, appartient à son cadre qui confère à la représentation iconique sa nature symbolique. C'est parce que l'animal n'a pas accès au symbolique, qu'il n'arrive pas à comprendre les images, même s'il perçoit parfaitement leur matérialité sensorielle et de ce fait reconnait leur représentation. Ce n'est donc pas la perception des ressemblances avec le représenté, mais le travail de mise en rapport des formes avec le monde auquel elles appartiennent qui est à l'origine de l'assignation du sens des images.

Il se peut évidemment que le sens d'une image nous échappe, mais ce n'est pas parce que son sens nous est inaccessible, ce n'est pas parce que notre mémoire n'arrive pas à reconnaître des segments d'expérience avec lesquels l'image établit des rapports qui puissent la situer, qu'elle n'a pas de sens pour nous. Ne pas identifier le sens d'une image ne veut pas dire qu'elle n'a pas de sens pour nous. Nous disons plutôt qu'elle acquiert alors un sens énigmatique.

Les présupposés de l'énonciation jouent dans le discours un rôle semblable à celui que le hors-champ joue pour les images fixées matériellement ${ }^{1}$. Telle l'image que je ne peux pas situer par rapport à son cadre, un énoncé demeurera énigmatique, même s'il est parfaitement compréhensible du point de vue de sa signification, si je n'arrive pas à le situer par rapport à son cadre énonciatif. Ainsi, c'est parce que je

1 O. DUCROT, Dire et ne pas dire, Paris, Hermann, 1991 (3ème édition). 
partage avec mes interlocuteurs, actuels ou virtuels, tout un ensemble de présupposés, des marques d'expériences mutuellement connues non dites, que peux comprendre le sens des paroles que nous partageons, des paroles qui reçoivent de ces présupposés ses raisons d'être énoncées. Partager des discours, c'est donc, en même temps, une question de reconnaissance d'un monde commun et une question de reconnaissance mutuelle et réciproque.

Le sens des images matériellement fixées ainsi que des images du discours font donc retourner des segments de l'expérience que la mémoire sélectionne du flux de mes expériences passées et que l'imagination constitue comme son cadre. Il se peut que les segments de l'expérience qui constituent le cadre du sens d'une image appartiennent à un vécu immédiat, qu'il soit prélevé d'images récentes qui n'ont pas encore subi les effets du travail de l'oubli. Quand je vois le déroulement des scènes d'un film ou lors d'une conversation, les images et les énoncés échangés demeurent disponibles pour assurer la continuité et la cohésion du sens de l'interaction dans laquelle je suis engagé. Le travail de montage et de disposition des raccords du film ou des images en mouvement, à l'instar des dispositifs anaphoriques et des marques de l'interaction des discours, se chargent d'entretenir en permanence la réactivation de la mémoire de ces expériences récentes qui constituent, maintiennent et rétablissent en permanence les frontières du cadre ou du monde auquel les images perçues appartiennent et dont elles reçoivent du sens. Mais il arrive aussi que ces frontières s'élargissent pour y faire rentrer des segments d'expériences éloignées dans le temps et/ou dans l'espace et que la perception, ici et maintenant, de l'image évoque et que l'imagination rapproche et fait retourner au présent. Dans ces cas c'est la frontière du monde imaginaire et, par conséquent, le cadre du sens qui s'élargit pour résorber ces fragments distants et les faire intervenir dans la compréhension, ici et maintenant, des images appréhendées.

Il est maintenant facile de comprendre ce qui différencie le sens des deux modalités d'images que je viens d'étudier du sens de l'image mentale. Souvenons-nous, tout d'abord, que les images matériellement fixées ainsi que les images du discours sont des produits d'un dispositif et gardent de cette origine des marques explicites ou implicites. De son côté, le cadre des images mentales est entièrement produit par le travail de mon imagination. Il est vrai que tant la contemplation d'images matériellement fixées que la perception d'images du discours engendrent aussi des images mentales, mais celles-ci ne se confondent pas avec les 
images contemplées sur le support matériel ou discursif. L'image que je contemple, par exemple, sur la photo trouvée sur mon bureau, l'image d'une annonce publicitaire, la suite d'images qui forment les planches de la bande dessinée, du reportage télévisé ou du film qui défile devant mes yeux sont autonomes par rapport à mon imagination et établissent avec elle un rapport d'extériorité, tandis que l'image mentale n'a pas de support extérieur à l'activité de mon imagination.

\section{Conclusion : la nature symptomatique du désir d'images dans le monde contemporain}

Cette distinction des images matériellement fixées et des images du discours par rapport aux images mentales a des conséquences pragmatiques importantes dont on doit aussi tenir compte. Du fait de leur immanence à l'expérience subjective, les images mentales échappent au travail d'objectivation d'un dispositif et ne peuvent donc pas être partagées à proprement parler. Je ne peux pas me rendre compte des images mentales des autres personnes qui, de leur côté, n'ont pas plus accès à mes propres images mentales. Par contre, du fait de l'extériorité et de l'autonomie que leur procurent le dispositif technique et le dispositif énonciatif, les images matérielles et les images discursives acquièrent une nature symbolique qui les destine ou les oriente vers l'échange au sein du monde intersubjectif. De ce fait, elles sont un des facteurs importants de la formation du lien social.

Etant donné la nature symbolique et le rapport au monde intersubjectif des images, je fais l'hypothèse que l'actuelle prolifération des images et l'invention de dispositifs de plus en plus performants peuvent être entendues comme l'expression du désir d'une modalité perdue de lien social et de reconnaissance. Ce désir accru d'images n'est-il pas un symptôme d'un manque ou d'une absence, d'une expérience perdue ? Ce désir, l'image le réalise de manière symbolique. Nous avons souligné les modalités de cette réalisation. La distinction et la mise en valeur de segments de l'expérience assure à l'image son référent, l'identification de la représentation. De son côté, la mise en rapport de l'image avec le monde imagétique lui assigne une valeur narrative et, de ce fait, lui assure un sens, ouvert au partage et au lien entre les partenaires du monde intersubjectif. Les images ne sont pas faites pour être gardées en secret. Même si elles peuvent être gardées pour soi comme souvenir ou comme preuve, elles ont comme destinée habituelle l'affichage et l'échange. 


\section{Bibliographie}

BERGSON, H., Essais sur les Données Immédiates de la Conscience, Paris, PUF, 2007 (original : 1888).

BERGSON, H., L'Evolution Créatrice, Paris, PUF, 2006 (édition originale : 1941).

COLLINS, R., Interaction Ritual Chains, Princeton and Oxford, Princeton University Press, 2005.

DELEUZE, G., Cinéma 1. L'Image-Mouvement, Paris, Minuit, 1983.

DELEUZE, G., Cinéma 2. L'Image-Temps, Paris, Minuit, 1985.

DUCROT, O. Dire et ne pas Dire, Paris, Hermann, 3ème édition, 1991.

GofFMAn, E., Les Cadres de l'Expérience, Paris, Minuit, 1977.

MEUNIER, J.-P., Essai sur l'Image et la Communication, Louvain-la-Neuve, Cabay, 1980.

MEUNIER, J.-P. \& PERAYA D., Introduction aux Théories de la Communication, Bruxelles, De Boeck-Wesmael, 1993.

SARTRE, J.-P., L'Imaginaire, Paris, Gallimard, 1940.

SCHÜTZ, A., The Phenomenology of the Social World, Northwestern University Press, 1967. 